\title{
eJRIEPS
}

Ejournal de la recherche sur l'intervention en éducation physique et sport

$20 \mid 2010$

Varia

\section{La transmission du savoir expérientiel en EPS : études de cas et analyses comparatives en didactique clinique}

Pablo Buznic-Bourgeacq, André Terrisse et Eric Margnes

\section{(2) OpenEdition}

1 Journals

Édition électronique

URL : http://journals.openedition.org/ejrieps/4784

DOI : $10.4000 /$ ejrieps.4784

ISSN : 2105-0821

Éditeur

ELLIADD

Référence électronique

Pablo Buznic-Bourgeacq, André Terrisse et Eric Margnes, "La transmission du savoir expérientiel en EPS : études de cas et analyses comparatives en didactique clinique », eJRIEPS [En ligne], 20 | 2010, mis en ligne le 01 avril 2010, consulté le 09 avril 2020. URL : http://journals.openedition.org/ejrieps/ 4784 ; DOI : https://doi.org/10.4000/ejrieps.4784

Ce document a été généré automatiquement le 9 avril 2020.

La revue eJRIEPS est mise à disposition selon les termes de la Creative Commons Attribution 4.0 International License. 


\title{
La transmission du savoir expérientiel en EPS : études de cas et analyses comparatives en didactique clinique
}

\author{
Pablo Buznic-Bourgeacq, André Terrisse et Eric Margnes
}

Ce texte rend compte d'une recherche menée en didactique de l'EPS, interrogeant le poids de l'expérience personnelle de pratiquant du professeur d'EPS dans une APSA spécifique sur le contenu de l'enseignement effectif de cette dernière (Buznic-Bourgeacq, 2009). Notre intention a été d'identifier ce poids de manière transversale, quelle que soit l'APSA enseignée. Nous avons donc émis une hypothèse simple: l'expérience de pratiquant dans n'importe quelle activité apporte quelque chose «en plus » au sujet enseignant qui peut alors, sous condition de cette expérience, transmettre quelque chose "en plus ».

Nous présentons alors ici le parcours de cette recherche et les éléments de connaissance qu'elle a pu apporter sur le poids de l'expérience personnelle du professeur d'EPS. Dans un premier temps, nous montrons comment la question de l'expérience personnelle du sujet enseignant nous a amené à construire une modélisation transversale du savoir expérientiel et de sa transmission. Nous présentons ensuite cette modélisation et son actualisation en outil d'analyse des pratiques d'enseignement effectif. Puis, après avoir présenté le cadre méthodologique qui nous a permis de mettre à l'épreuve cet outil d'analyse, nous exposons la démarche progressive de construction des résultats que nous avons effectuée. Enfin, au travers d'interprétations propres à notre posture épistémologique, la didactique clinique (Terrisse, Carnus, 2009), nous présentons les éléments de conclusion auxquels nous en sommes arrivés au terme de cette recherche, concernant les rapports entre expérience personnelle et enseignement effectif. 


\section{L'expérience personnelle de l'enseignant : d'une problématique de recherche à la construction d'un cadre d'analyse des pratiques d'enseignement}

\section{1. La problématique de l'expérience personnelle du professeur d'EPS}

2 La question du poids de l'expérience personnelle du professeur d'EPS sur ses pratiques d'enseignement trouve son origine dans une situation socioprofessionnelle singulière et problématique. En effet, les enseignants débutants en EPS font leurs premiers pas dans l'enseignement scolaire en ayant à enseigner une multiplicité d'APSA. Dans cette diversité, ils se confrontent alors à l'enseignement d'activités dans lesquelles ils n'ont aucune expérience personnelle de pratiquant.

L'intuition peut aisément permettre d'entrevoir l'ambiguïté de cette situation. Nous avons voulu, en tant que didacticiens, l'interroger de manière théorique et empirique afin de déterminer quels pouvaient être les manques pour l'enseignant à défaut d'expérience personnelle dans l'activité enseignée. De manière symétrique, nous avons aussi voulu caractériser quels pouvaient être les apports de l'expérience personnelle de pratique pour le professeur d'EPS débutant.

Les recherches en EPS ont très peu questionné ces apports et ces manques relatifs à l'expérience personnelle des enseignants. Comme si cette expérience subjective allait de soi, c'est davantage le poids de l'expérience professionnelle d'enseignement auquel les chercheurs se sont intéressés (Carnus, Garcia-Debanc, Terrisse, 2008). Quelques travaux se sont toutefois penchés sur les rapports entre les connaissances des enseignants et leur expérience personnelle de pratiquant(Soler, Durand, 1998; Piéron, 1998). Ces recherches ont ainsi montré que l'expérience personnelle de l'enseignant lui a permis de construire des connaissances disciplinaires davantage profondes et articulées et une capacité de repérage des erreurs des pratiquants plus efficace. Mais ces études demeurent des recherches de laboratoires qui n'affrontent pas les pratiques effectives d'enseignement. Nous avons alors voulu initier ce travail en allant identifier les ressorts de l'expérience personnelle dans les pratiques effectives.

Pour identifier ces apports et ces manques, nous avons alors choisi d'adopter un regard spécifique sur les pratiques d'enseignement. Nous avons voulu identifier le poids de l'expérience personnelle sur le contenu des processus de transmissions des savoirs en EPS. Nous avons donc cherché à identifier ce qui était enseigné lorsque l'enseignant témoignait ou non d'une expérience personnelle de pratiquant. Cette tentative d'identification a alors été menée dans une perspective générique, c'est-à-dire en ayant la volonté de caractériser le poids de l'expérience personnelle sur le contenu des pratiques quelle que soit l'activité enseignée, quel que soit le savoir enjeu de la transmission.

Cela nous a amené à établir une question de recherche transversale: qu'est-ce que l'expérience personnelle permet à l'enseignant de transmettre «en plus»?

\section{2. L'en plus de savoir issu de l'expérience}

3 Cet « en plus » constitue notre objet de recherche. Il ouvre ainsi la réflexion théorique à partir d'un double postulat. Tout d'abord, celui de l'existence d'un «en plus» de savoir 
construit dans l'expérience par le sujet, quelle que soit l'activité dans laquelle se déploie cette expérience. Sans parler encore ici de transmission des savoirs, nous postulons qu'il existe une part du savoir qui n'appartient qu'à l'expérience, qu'au sujet expérimenté. Cet "en plus» de savoir est donc générique à toute expérience, mais spécifique à l'expérience personnelle de pratique. Ensuite, nous postulons l'existence d'un processus de «conversion didactique » (Buznic-Bourgeacq, 2005) du contenu de l'expérience en contenu de l'enseignement. Dans une approche clinique de la transposition didactique, nous considérons que les éléments de savoir construits par l'enseignant dans son expérience personnelle vont organiser le contenu de ses pratiques effectives d'enseignement. Ainsi, l' "en plus» de savoir construit dans l'expérience est ici considéré comme un organisateur du contenu des pratiques.

La construction de notre cadre théorique a donc consisté en deux temps. Nous nous sommes tout d'abord attachés à caractériser cet «en plus ». Nous l'avons ainsi défini en termes de savoir expérientiel, c'est-à-dire un savoir spécifiquement construit dans l'expérience de pratique, mais générique à toute expérience dans n'importe quelle activité. Cette étape a ainsi consisté en une analyse épistémologique de l'expérience, afin de dégager théoriquement les dimensions transversales, génériques, propres au savoir expérientiel. Nous avons alors ensuite mené une analyse transpositive théorique de ces dimensions transversales afin d'identifier de quelle manière elles pouvaient organiser le contenu des processus de transmission.

Ce travail théorique nous a alors menés à la construction d'un cadre d'analyse des pratiques effectives d'enseignement.

\section{Les dimensions constitutives du savoir expérientiel organisant le contenu des pratiques effectives}

\section{1. Analyse épistémologique de l'expérience : les dimensions constitutives du savoir expérientiel}

4 Pour caractériser le savoir expérientiel et ses dimensions constitutives, nous avons effectué un parcours théorique entre les didactiques, les sciences de l'éducation, les sciences cognitives, l'épistémologie et la philosophie de la connaissance. Notre intention a été, par une approche transdisciplinaire, de dégager la part spécifique du savoir construit par le sujet dans son expérience personnelle et de la modéliser sous la forme d'une organisation systémique entre plusieurs dimensions constitutives.

Nous avons alors procédé en quatre étapes. Dans un premier temps, nous avons tenté de «situer » le savoir d'une manière générale. Car le savoir n'est pas un objet isolé ou autonome, nous l'avons d'abord caractérisé dans ses relations avec le réel (Bachelard, 1938; Juranville, 1978), les sujets (Beillerot, 1996) et les institutions (Durkheim, 1912 ; Sensevy, 1997). Ayant identifié le système dans lequel le savoir évolue, nous nous sommes ensuite penchés sur une part spécifique du savoir. Dans la mesure où l'expérience personnelle dans une activité renvoie en grande partie à une expérience pratique, nous nous sommes intéressés aux spécificités du savoir généré dans la pratique.

Nous avons principalement retenu sa dimension singulière, dynamique et situationnelle (Tochon, 1996), son organisation global et syncrétique (Bourdieu, 1980 ; Vigarello, Vives, 1986) et son appartenance à l'initiative du sujet (Tochon, 1996). 
À partir de ces spécificités, nous avons par la suite examiné les problématiques qui nous semblaient les plus fondamentales à propos du savoir expérientiel, pour tenter par alors de trouver des systèmes théoriques capables de les prendre en compte et de proposer du contenu à ce savoir expérientiel. Trois problématiques ont été identifiées, nommées celle du sujet, celle du réel et celle de l'immédiateté de leur relation. Elles nous ont permis de caractériser le savoir expérientiel comme un savoir d'initiative (Schlanger, 1978), un savoir finalisé (Vergnaud, 1996 ; Récopé, 1996), un savoir constitué d'invariances pour identifier et catégoriser le réel (Vergnaud, 1994,1996), un savoir global (Piaget, 1936; Wallon, 1942, Vergnaud, 2001), un savoir formé de processus inférentiels nécessaire pour appréhender des situations singulières (Beillerot, 1996 ; Vergnaud, 1985).

Ces différents éléments propres au savoir expérientiel nous ont ainsi amenés vers le concept de schème, principalement tel qu'il est théorisé par Vergnaud (1985, 1994, 1996, 2001). À partir des quatre composants analytiques du schème qu'il définit, les buts, les règles, les invariants opératoires et les processus inférentiels, nous avons proposé un contenu à une dimension spécifique du savoir expérientiel, sa dimension conceptuelle. La modélisation a donc été menée au niveau du traitement cognitif des situations, au niveau des représentations conceptuelles qui servent d'intermédiaire nécessaire pour organiser les conduites du sujet en situation. Nous avons alors voulu, pour compléter la modélisation, analyser ce qui pouvait donner du contenu au savoir expérientiel au-delà du traitement cognitif des situations. Nous avons alors mené une double ouverture, pour aller voir au-delà du cognitif, en nous intéressant aux inscriptions institutionnelle et corporelle de l'expérience. Nous avons alors identifié la dimension institutionnelle du savoir expérientiel, au travers du savoir stabilisé et reconnu par l'intermédiaire des signifiants (Wallon, 1942 ; Vergnaud, 1987 ; Brun, 1996) et sa dimension corporelle, au travers du savoir incorporé dans des sensations et des repères sensoriels (Varela, 1993 ; Merleau-Ponty, 1945 ; Sherrington, 1933).

Enfin, à partir de ce parcours théorique, nous avons tenté d'organiser ces différents éléments sous la forme de dimensions constitutives du savoir expérientiel, articulées entre elles de manière systémique, mais se prêtant à l'analyse dans une perspective d'identification empirique.

Nous avons alors pu dégager six dimensions constitutives du savoir expérientiel : la dimension conceptuelle, la dimension signifiante, la dimension corporelle, la dynamique situationnelle / représentationnelle, la part d'initiative et l'organisation globale.

Nous n'explicitons pas ici ces dimensions en dehors de leur inscription didactique, dans la mesure où leur identification n'est bien qu'une étape dans la construction de notre cadre d'analyse des pratiques d'enseignement. L'étape suivante a donc consisté à mener une analyse transpositive théorique de chacune de ces six dimensions, afin d'identifier de quelle manière elle peut organiser le contenu des processus de transmission des savoirs.

\section{2. Analyse transpositive du savoir expérientiel : construction d'un cadre d'analyse des pratiques}

5 L'intention est ici de caractériser l'inscription didactique des six dimensions constitutives du savoir expérientiel, afin de dégager différents éléments révélateurs de ces dimensions dans les pratiques d'enseignement effectif. L'identification de ces 
éléments sous la forme d'indicateurs empiriques constitue alors le projet de cette démarche, établie dans une perspective de construction d'un cadre d'analyse des pratiques.

Pour établir ces indicateurs empiriques, nous avons identifié, pour chaque dimension, à partir de quels éléments elle organise le contenu des pratiques d'enseignement et quels sont les vecteurs didactiques de ces éléments. Nous entendons par «vecteurs didactiques ", les différents médias qui permettent à l'enseignant de transmettre des éléments de savoir: une situation adidactique, un discours, une démonstration corporelle, etc. Nous les présentons dans le tableau 1 pour offrir une vision synthétique de notre cadre d'analyse.

Tableau I. Les six dimensions constitutives du savoir expérientiel organisant le contenu des pratiques d'enseignement

\begin{tabular}{|c|c|c|}
\hline $\begin{array}{l}\text { Les } 6 \text { dimensions } \\
\text { constitutives du savoir } \\
\text { expérientiel }\end{array}$ & Un contenu organisé par... & $\begin{array}{l}\text { Vecteurs } \\
\text { didactiques }\end{array}$ \\
\hline La dimension conceptuelle & $\begin{array}{l}\text { Des invariants opératoires, articulés entre } \\
\text { eux sous la forme de hiérarchies de buts et de } \\
\text { règles d'action }\end{array}$ & \begin{tabular}{lr}
- & \multicolumn{2}{c}{ Situations } \\
adidactiques & \\
- & Discours \\
l'enseignant & de \\
- & \multicolumn{2}{r}{ Procédures } \\
ostensives r non \\
verbales r
\end{tabular} \\
\hline La dimension signifiante & $\begin{array}{l}\text { Des signifiants appartenant à des champs } \\
\text { lexicaux spécifiques (APSA ou autres) }\end{array}$ & $\begin{array}{l}-\quad \text { Discours de } \\
\text { l'enseignant }\end{array}$ \\
\hline La dimension corporelle & Des sensations et des repères sensoriels & $\begin{array}{l}\text { - Situations } \\
\text { adidactiques } \\
\text { - Démonstrations } \\
\text { - Manipulations } \\
\text { - Attentions } \\
\text { sensorielles }\end{array}$ \\
\hline $\begin{array}{l}\text { La dynamique } \\
\text { situationnelle / } \\
\text { représentationnelle }\end{array}$ & $\begin{array}{l}\text { Des processus inférentiels qui articulent } \\
\text { chaque invariant dans des situations } \\
\text { singulières et dans des représentations }\end{array}$ & $\begin{array}{l}\text { - Discours analogique } \\
\text { de l'enseignant } \\
\text { - Enchaînements des } \\
\text { situations } \\
\text { adidactiques }\end{array}$ \\
\hline La part d'initiative & $\begin{array}{l}\text { Des choix potentiels dans la définition des } \\
\text { tâches et dans les interactions }\end{array}$ & $\begin{array}{l}\text { - Discours de } \\
\text { l'enseignant }\end{array}$ \\
\hline L'organisation globale & $\begin{array}{l}\text { Une organisation globale qui se distingue de } \\
\text { la somme des différents éléments }\end{array}$ & $\begin{array}{l}\text { - Situations } \\
\text { adidactiques }\end{array}$ \\
\hline
\end{tabular}


En allant analyser empiriquement différentes pratiques d'enseignement en EPS, ce cadre d'analyse nous a alors permis d'identifier des organisations spécifiques du contenu de ces pratiques, au regard des expériences personnelles singulières dont témoignaient les enseignants dans les APSA enseignées.

\section{Méthodologie : des expériences contrastées, une démarche progressive d'analyse}

\section{1. Terrain empirique}

7 Le choix du terrain de notre recherche s'est fondé sur la volonté de comparer des pratiques d'enseignement menées par des enseignants ayant des expériences personnelles contrastées, prolongée ou inexistante, dans les activités enseignées. Nous avons alors choisi de travailler avec quatre professeurs d'EPS débutants, n'ayant donc aucune expérience professionnelle dans l'enseignement et enseignants chacun deux ou trois APSA dans lesquelles ils ont une expérience prolongée (plus de cinq ans de pratique extrascolaire) ou inexistante (aucune pratique extrascolaire). Le choix d'enseignants débutants pour étudier le poids de l'expérience personnelle permet ainsi d'analyser leur activité didactique sans que celle-ci ne soit déterminée par une expérience professionnelle d'enseignement. Tous les enseignements sont menés face à des élèves de $6^{\text {ème }}$ ou du cycle central dans des collèges publics de la région Aquitaine.

Tableau II. Cadre empirique de la recherche

\begin{tabular}{|l|l|l|l|l|l|}
\hline & Basket & Rugby & Natation & Danse & Gymnastique \\
\hline P1 & & Ex & Ex & & NEx \\
\hline P2 & NEx & Ex & & & \\
\hline P3 & NEx & & & Ex & \\
\hline P4 & NEx & & Ex & NEx & \\
\hline
\end{tabular}

EX : Expérience prolongée dans l'APSA. NEX : Aucune d'expérience dans l'APSA

8 L'analyse comparative de ces différents enseignements permet ainsi d'effectuer plusieurs croisements intra et intersubjectifs pour dégager pas à pas le poids de l'expérience personnelle sur les pratiques effectives.

\section{2. Recueil des données}

9 Pour chacun de ces enseignements, nous avons mené des observations vidéo de trois séances consécutives (séances 2 , 3 et 4 de chaque cycle). Ces observations ont été entièrement retranscrites. Les différents indicateurs empiriques ont donc été identifiés à partir de ces retranscriptions.

Ensuite, pour chaque séance observée, nous avons aussi mené un entretien post-séance au travers duquel nous avons interrogé chaque enseignant sur ses intentions 
didactiques, les situations mises en places (et leurs liens avec ses intentions didactiques), les émergences de savoir in situ (qu'il n'avait pas prévus a priori) et sur ses préoccupations subjectives en classe (ce qu'il vient de vivre et de ressentir). Ces entretiens nous ont ainsi permis de croiser notre point de vue externe de chercheur sur les pratiques avec leur point de vue interne de sujet enseignant.

Enfin, nous avons mené avec chaque enseignant un entretien d'«aprèscoup » (Terrisse, 2000). Au travers de cet entretien, effectué plusieurs mois après les observations, nous avons voulu avoir accès aux descriptions et interprétations des enseignants sur leurs propres pratiques. En les interrogeant sur les spécificités qu'ils avaient pu percevoir dans leurs différents enseignements, nous avons pu interpeler leur point de vue subjectif sur le poids de leur expérience et identifier les causes qu'ils attribuent à leurs propres descriptions.

\section{3. Démarche progressive d'exploitation des données}

À partir de cet ensemble de données et des indicateurs empiriques préalablement construits, nous avons établi une démarche d'exploitation en trois temps, dans le but d'identifier progressivement le poids de l'expérience personnelle sur le contenu des pratiques effectives.

Tout d'abord, nous avons mené un ensemble de comparaisons macroscopiques sur l'ensemble des enseignements observés. Nous avons comparé quantitativement, pour chaque indicateur empirique relatif à chaque dimension, les moyennes entre l'ensemble des cinq enseignements avec expérience et l'ensemble des cinq enseignements sans expérience. Cette étape nous a alors permis de dégager des tendances génériques propres à chaque ensemble d'enseignement, c'est-à-dire déterminées par le poids de l'expérience personnelle. Le traitement quantitatif des données s'appuie ici simplement sur une comparaison de moyennes. La volonté de cette étape quantitative résidant dans l'identification de tendances génériques préalables à l'analyse clinique, la population choisie s'avère limitée à quatre individus. Bien que quantitative, cette étape ne prétend alors pas à une analyse statistique inférentielle, mais bien à une exploration descriptive.

Ensuite, dans la mesure où ces comparaisons macroscopiques ne prennent pas en compte la spécificité des APSA enseignées ni la singularité des sujets enseignants, nous avons poursuivi l'exploitation des données à partir d'analyses qualitatives. En nous arrêtant sur les résultats les plus significatifs au niveau quantitatif (toujours identifiés à partir de nos indicateurs empiriques), nous avons caractérisé les organisations singulières du contenu de chaque enseignement, relativement à chaque dimension. Nous avons donc tenté d'identifier ce qui dans le contenu et la forme des processus didactiques, pouvait déterminer ces résultats significatifs; cela sans encore apporter d'interprétations extérieures aux pratiques observées. Ainsi, au fil des analyses qualitatives, nous avons fait de nombreux allers-retours interprétatifs entre les six dimensions. Ce qui nous a permis d'effectuer de très nombreuses relectures de chaque enseignement et de progressivement décloisonner le cadre d'analyse initial pour identifier des problématiques propres à chaque enseignant qui organisent le contenu de ses pratiques. Cette étape nous a alors permis de construire progressivement le cas de chaque enseignant, c'est-à-dire d'identifier comment l'expérience personnelle pèse spécifiquement chez chaque enseignant.

Cette première perspective clinique, qui s'attache à construire le cas de chaque sujet 
enseignant au-delà des comparaisons, a ainsi amorcé le dernier temps de la démarche d'exploitation des données. En effet, nous avons ensuite analysé le discours des enseignants sur leurs propres pratiques, issu des entretiens post-séances et d'aprèscoup. À partir des problématiques dégagées dans l'analyse des pratiques pour construire le cas de chaque enseignant, nous avons mené une analyse thématique du contenu de ces entretiens (Poussin, 2003). Nous nous sommes alors particulièrement arrêtés sur le discours interprétatif des enseignants. Cette analyse a été effectuée au travers de cadres interprétatifs propres à la didactique clinique (Terrisse, Carnus, 2009). Nous en exposons la spécificité simultanément à la présentation des cas.

\section{Résultats et interprétations : trois temps pour identifier le poids de l'expérience}

\section{1. Comparaisons quantitatives : tendances génériques}

11 Nous présentons ici une explicitation synthétique des tendances génériques, pour chaque dimension, des deux ensembles d'enseignements (Ex et NEx) comparés quantitativement. Les résultats quantitatifs sont présentés en annexe dans le tableau 3.

\section{1. 1. La dimension conceptuelle}

12 Nous identifions ici les invariants opératoires mis en jeu par les enseignants en classe. Ces invariants sont les objets, les relations, les propriétés que le sujet découpe dans le réel pour organiser ses conduites (Vergnaud, 1989). Nous considérons qu'en classe, pour enseigner, le professeur découpe l'APSA enseignée en différents invariants (par exemple en rugby, "le soutien du non porteur ", «le plaquage », " fixer la défense ", etc.). Nous avons alors identifié la diversité et la redondance de ces invariants dans chaque enseignement. Mais, l'enseignant peut aussi mettre en jeu ces invariants de manière plus ou moins articulée, sous la forme de hiérarchies de buts ("fixer la défense » pour «créer des espaces libres») et de règles d'action (si « on passe » alors « on se replace»). Nous avons aussi identifié ces articulations.

Ainsi, le contenu des enseignements Ex est beaucoup plus dense que celui des NEx. Les enseignants y ont tendance à découper l'APSA en une plus grande diversité d'invariants opératoires (plus d'une fois et demie). De plus, ils mettent beaucoup plus d'invariants en jeu de manière articulée, sous la forme de hiérarchies de buts (plus de trois fois) et de règles d'action (plus de quatre fois). Le contenu des enseignements Ex est donc organisé à partir d'une plus grande diversité d'objets davantage articulés entre eux. La structure conceptuelle qui organise ce contenu s'avère donc beaucoup plus dense.

\section{1. 2. La dimension signifiante}

Nous identifions ici les signifiants mis en jeu par les enseignants. Dans le processus de transmission, l'enseignant use de signifiants (Vergnaud, 1987), c'est-à-dire d'unités langagières, pour conceptualiser ou contextualiser l'APSA enseignée. Ces signifiants se distinguent des invariants opératoires dans la mesure où ils appartiennent au langage 
et sont donc par nature arbitraires (Wallon, 1942). Ainsi, ils ne peuvent être crées par le sujet, ils lui préexistent au sein d'un code commun. Les signifiants appartiennent alors en premier lieu davantage aux institutions qu'au sujet. On peut donc identifier des institutions spécifiques auxquelles chaque signifiant fait référence, comme notamment l'APSA enseignée (par exemple en rugby, "la mêlée ", "le maul ", etc.). Nous avons donc identifié dans chaque enseignement la diversité des signifiants mis en jeu par l'enseignant pour conceptualiser l'APSA enseignée et leur appartenance institutionnelle, c'est-à-dire la diversité des signifiants appartenant à telle ou telle institution spécifique, notamment celle de l'APSA enseignée.

Ainsi, dans les enseignements Ex, l'activité de verbalisation des enseignants est beaucoup plus dense, mais aussi beaucoup plus spécifique. Les enseignants y utilisent davantage de signifiants distincts pour conceptualiser l'APSA enseignée (plus d'une fois et demie) que dans les NEx. Ils ont donc beaucoup plus recours à la verbalisation pour enseigner, mais surtout ils ont recours à une verbalisation plus spécifique. Ils utilisent davantage de signifiants propres à l'APSA enseignée (près de deux fois et demie).

\section{1. 3. La dimension corporelle}

14 Nous identifions ici les sensations et repères sensoriels mis en jeu par les enseignants. Il y a une part du savoir qui échappe à la conceptualisation et encore davantage à l'explicitation. Il s'agit des sensations. Pour autant nous postulons que l'enseignant peut transmettre ces «états bruts et immédiats» (Lalande, 1926) au travers de procédures didactiques spécifiques : certaines situations adidactiques, la démonstration corporelle, la manipulation du corps de l'élève ou l'attention sensorielle. Nous avons donc identifié dans chaque enseignement l'intensité du recours à ces procédures. Mais l'enseignant peut aussi transmettre des repères sensoriels qui, eux, passent par la conceptualisation. Nous avons donc aussi identifié la multiplicité des repères sensoriels extéroceptifs et proprioceptifs mis en jeu par les enseignants en classe.

Ainsi, dans le contenu des enseignements Ex, le corps est beaucoup plus présent. Les enseignants y mettent en jeu beaucoup plus de repères sensoriels extéroceptifs (près de deux fois) et proprioceptifs (plus de deux fois) que dans les NEx. Ils ont tendance à arrêter plus fréquemment l'activité perceptive des élèves sur les états et les modifications de l'environnement ou de leur propre corps. De plus, les procédures didactiques vectrices de sensations sont beaucoup plus utilisées dans les Ex. Particulièrement, la démonstration corporelle demeure le privilège de ces enseignements (plus de cinq fois). Les enseignants $\mathrm{y}$ ont ainsi tendance à pénétrer davantage le milieu de l'élève corporellement.

\section{1. 4. La dynamique situationnelle/représentationnelle}

Nous identifions ici les processus inférentiels mis en jeu par les enseignants. Ces processus renvoient à la capacité du sujet à articuler les invariants dans différentes situations singulières et à extraire ces invariants de la singularité de chaque situation pour les inscrire dans des représentations génériques. Dans le processus de transmission, l'enseignant va alors pouvoir favoriser ces processus inférentiels en permettant aux élèves de réinvestir les différents invariants dans des situations plus ou moins distinctes. Nous avons donc identifié les enchaînements de situations adidactiques proposés par chaque enseignant, en suivant à la trace chaque invariant 
sous-jacent à chaque situation. Nous nous sommes alors arrêtés sur la forme de ces réinvestissements d'invariants: à l'identique, directs, explicites, implicites. Mais l'enseignant peut aussi favoriser les processus inférentiels dans son discours, au travers d'analogies. Nous avons donc aussi identifié les nombre d'analogies utilisées par chaque enseignant, en distinguant les analogies internes à l'institution classe (par exemple, "c'est comme la semaine dernière ») et les analogies externes (par exemple, «c'est comme à la télé »).

Ainsi, dans les enseignements Ex, les processus inférentiels sont largement valorisés. Tout d'abord, on assiste à davantage de réinvestissements d'invariants entre les situations (plus d'une fois et demie) que dans les NEx. Cela car les situations proposées par les enseignants sont organisées à partir d'un plus grand nombre d'invariants sousjacents. Mais ces réinvestissements prennent aussi des formes spécifiques. Dans les Ex, ces réinvestissements sont plus directs et plus explicites. D'une part, les situations s'enchaînent de manière plus progressive ; les situations successives renvoient souvent à des réaménagements succincts, à partir d'une ou deux variables, des situations qui les précèdent. D'autre part, les réinvestissements sont plus explicites, dans la mesure où les enseignants explicitent les rapports entre les différentes situations. Cela au travers d'analogies internes bien plus nombreuses (plus de deux fois et demie) que dans les NEx. Le contenu des enseignements Ex est donc plus dynamique puisque les différents invariants mis en jeu s'articulent davantage dans des espaces-temps enchevêtrés.

\section{1. 5. La part d'initiative}

16 Nous identifions ici les choix potentiels mis en jeu par les enseignants. Le savoir expérientiel répond à l'initiative du sujet. Il ne se réfère pas à une autorité extérieure, sa construction et sa mise en œuvre sont sous la responsabilité du sujet. En classe, l'enseignant peut alors valoriser cette initiative au travers des choix potentiels laissés aux élèves. Lorsque l'enseignant définit explicitement les tâches qui incombent aux élèves, il peut plus ou moins laisser aux élèves l'initiative des moyens à mettre en œuvre pour atteindre les buts déterminés. En analysant ces phases de définition (Sensevy, Schubauer-Léoni, Mercier, 2000), nous avons alors identifié le nombre de tâches dans lesquelles les choix potentiels sont illimités pour les élèves (par exemple en natation, "il faut réduire les cycles de bras») ou au contraire inexistants (par exemple, «il faut réduire les cycles de bras, donc vous devez faire des mouvements de bras lents, rester droits et faire des battements de jambes actifs »).

Ainsi, dans les enseignements Ex, les enseignants verrouillent davantage les choix potentiels pour les élèves. On peut identifier plus de situations dans lesquelles les élèves n'ont aucun choix potentiel (une fois et demie) que dans les NEx. Les enseignants $\mathrm{y}$ ont tendance à donner aux élèves, dès les phases de définition, les solutions efficaces pour atteindre les buts des tâches proposées. On assiste alors dans ces enseignements à un glissement des moyens à mettre en œuvre en buts à atteindre. Les solutions efficaces pour atteindre les buts sont devenues elles-mêmes des buts organisateurs et ne laissent plus de choix aux élèves. 


\section{1. 6. L'organisation globale}

17 Nous identifions ici la globalité des situations mises en place par les enseignants. Le savoir expérientiel se construit de manière globale, c'est-à-dire qu'il n'est pas sectorisé, qu'il forme un tout organisé dans lequel chaque élément ne prend sens que par rapport à l'ensemble des autres éléments. Ainsi, dans le processus de transmission, l'enseignant peut plus ou moins respecter cette globalité. Il peut notamment proposer des situations aux élèves plus ou moins proches des situations de pratique globale de l'APSA (par exemple en rugby, un match). Nous avons donc identifié, pour chaque enseignement, le nombre de situations globales, fragmentées et aménagées mises en place.

Ainsi, dans les enseignements Ex, les enseignants respectent davantage la globalité de l'APSA enseignée. Notamment, ils proposent beaucoup plus de situations de pratique globale (près de trois fois) que dans les NEx. Ils ont tendance à beaucoup moins didactiser les situations, c'est-à-dire à y insérer moins de variables didactiques artificielles.

Tous ces résultats comparatifs montrent déjà combien l'expérience personnelle de l'enseignant pèse sur l'organisation $d u$ contenu des pratiques effectives d'enseignement. Nous proposerons en conclusion une synthèse articulée de ces différents résultats propres à chaque dimension. Mais, comme nous l'avons dit, nous nous sommes attachés à dépasser ces comparaisons macroscopiques dans notre démarche d'exploitation des données.

Pour prendre en compte la spécificité des APSA enseignées et la singularité des sujets enseignants, nous avons mené des analyses qualitatives des pratiques.

\section{2. Analyses qualitatives: des organisations singulières du contenu}

18 Nous avons explicité la trame générale de notre démarche d'analyse qualitative. L'aboutissement de cette démarche nous a conduits à construire le cas de chaque enseignant face au poids de l'expérience personnelle. Nous présentons ici deux exemples, relatifs à deux cas, $\mathrm{P} 1$ et $\mathrm{P} 4$, dans leurs enseignements sans expérience. Nous voulons montrer comment notre démarche clinique nous a permis d'identifier des organisations singulières du contenu propres à chaque enseignant. Nous exposons alors ici plusieurs problématiques spécifiques organisant le contenu des pratiques de deux enseignants, dès lors qu'ils ne peuvent s'appuyer sur une expérience prolongée dans l'APSA enseignée. Dans ce texte, le choix d'une présentation des cas de P1 et P4 dans leurs enseignements sans expérience est justifié à deux niveaux. D'une part, l'arrêt sur des enseignements sans expérience nous permet de revenir sur le questionnement originel de notre recherche : celui relatif à la situation troublante des professeurs d'EPS débutants. D'autre part, le contenu des enseignements de P1 et P4 s'organise de manière très différente et permet ainsi de mettre en avant la singularité du poids de l'expérience personnelle chez chaque sujet enseignant.

\section{2. 1. P1 : les « allures » de l'expérience et la division du corps de l'enseignant}

$19 \mathrm{Au}$ regard des tendances génériques préalablement identifiées, l'enseignement NEx (gymnastique) mené par P1 prend des «allures » 
d'un enseignement Ex. D'un point de vue conceptuel, le contenu de son enseignement est relativement dense. Il intervient beaucoup auprès des élèves et enseigne à partir d'une diversité d'invariants relativement articulés entre eux. De même, dans le contenu de cet enseignement, le corps est très présent. P1 transmet un bon nombre de sensations et de repères sensoriels. Par-dessus tout, contrairement aux autres enseignements NEx, P1 s'engage dans des démonstrations corporelles relativement nombreuses.

Toutefois, si P1 s'engage corporellement même à défaut d'expérience, ces « allures » de l'expérience ne peuvent véritablement tenir sans embarras. En effet, on assiste dans cet enseignement à ce que nous avons appelé une division du corps de l'enseignant. Si P1 souhaite démontrer et démontre aux élèves, pour autant le réel de son incompétence corporelle en gymnastique va progressivement prendre le dessus. Dans un premier temps, lorsque P1 démontre, c'est en faisant l'aveu de son incompétence, par exemple en disant aux élèves : "bon là c'était nul, d'accord, je ne suis pas du tout monté à l'ATR »(S1) ou " honnêtement, je sais plus le faire là... enfin je vais essayer » (S1). Puis, au fur et à mesure des séances, ces aveux vont devenir des excuses. P1 va s'excuser de cette incompétence : "je suis désolé de pas pouvoir vous le démontrer»(S2). Puis, à partir de la deuxième séance, il va progressivement arrêter les démonstrations en s'excusant de s'être blessé en faisant une démonstration en classe (" aujourd'hui je ferai pas de démonstration, je me suis fait mal en faisant l'ATR roulé tout nul»), puis la veille à l'entraînement ("je vous jure, je me suis blessé lundi soir, donc je ferai aucune démo »). On assiste donc à une diminution progressive du recours aux démonstrations qui va le conduire à l'abandon total de cette procédure. Cet abandon va alors l'amener finalement à un abandon complet de l'enseignement de la gymnastique, puisqu'au milieu de la troisième séance, il arrête la gymnastique pour partir faire du handball.

\section{2. 2. P4 : un contenu en arrière-plan et l'absence du corps}

Contrairement à P1, dans les enseignements NEx menés par P4 (danse et basket), le contenu demeure en arrière-plan. Celui-ci a tendance à n'y enseigner qu'au travers des situations adidactiques. Il s'attache en permanence à aménager lourdement le milieu de l'élève à partir de variables didactiques. Ce milieu est donc très didactisé et les contraintes didactiques très marquées. Par exemple, en basket, pour enseigner " la progression vers la cible », il en passe par un jeu appelé «basket-rugby » couplé avec un système d'observation. En danse pour enseigner « la transmission de sensations à un public ", il demande d'abord aux élèves d'imiter des animaux; ce qui sera un prétexte à l'expression de verbes d'action qui sera enfin un prétexte à la transmission de sensations. Son enseignement passe donc presque uniquement par les aménagements didactiques. Il verbalise alors très peu le contenu et son activité de verbalisation porte davantage sur l'organisation didactique des situations que sur le contenu lui-même (par exemple sur le fonctionnement du système d'observation ou sur les prétextes didactiques).

Toujours d'une manière très différente de $\mathrm{P} 1$, dans le contenu des enseignements NEx menés par $\mathrm{P} 1$, le corps est particulièrement absent. Il transmet très peu de sensations et de repères sensoriels. Surtout, contrairement à $\mathrm{P} 1$, il ne s'engage presque jamais dans des démonstrations corporelles. Son corps ne porte jamais le savoir et ne constitue pas une part du milieu de l'élève.

La présentation succincte de ces deux cas montre qu'au-delà des tendances génériques 
du poids de l'expérience, le contenu des pratiques de chaque enseignant s'organise singulièrement à défaut d'expérience. D'un côté, P1 intervient largement d'un point de vue didactique et s'engage dans de nombreuses démonstrations corporelles dont l'ambiguïté le conduit à l'abandon. De l'autre, P4 n'enseigne que derrière ses aménagements didactiques et ne se risque pas à l'engagement corporel.

Nous voudrions alors montrer, au travers de notre troisième temps de la démarche d'exploitation, comment l'analyse du discours des enseignants sur leurs propres pratiques, interprété au travers de cadres propres à la didactique clinique, permet d'entrevoir, derrière ces singularités, une structure générique organisatrice des pratiques.

\section{3. Construction de cas : le sujet didactique et le poids de l'expérience}

21 Dans cette étape de l'analyse, nous nous sommes arrêtés sur le discours interprétatif des enseignants, relativement aux problématiques dégagées dans l'analyse des pratiques. L'intention a été d'identifier les « enjeux du sujet didactique " (BuznicBourgeacq, Terrisse, Lestel, 2008). Il s'agit d'une approche spécifique des pratiques, propre à la didactique clinique. On considère en effet que dans le didactique, le sujet enseignant est animé par des enjeux qui dépassent les enjeux de transmission, les enjeux proprement didactiques. Il est aussi animé par des enjeux subjectifs, principalement d'affirmation ou de sauvegarde de sa position spécifique dans le didactique. Ces enjeux vont donc être des organisateurs de l'activité didactique de chaque enseignant.

Pour interpréter ces enjeux, nous nous sommes appuyés sur des cadres interprétatifs spécifiques que nous présenterons simultanément aux cas de P1 et P4. Cette présentation simultanée est justifiée par la démarche clinique sous-jacente à la construction de ces cadres. En effet, c'est seulement à partir des cas qu'une structure théorique a pu être bâtie. Retenons toutefois qu'ils s'inscrivent dans un projet conceptuel de la didactique clinique qui consiste à construire une « théorie du sujet didactique » (Terrisse, Carnus, 2009), c'est-à-dire une théorie du jeu de codéterminations qui s'instaure entre le sujet, définit par ses enjeux subjectifs, et le didactique, définit par ses assujettissements singuliers (Buznic-Bourgeacq, 2009).

\section{3. 1. P1, la tentative d'illusion et l'échappée : position symbolique, position réelle et compromis}

P1 nous livre de front ce qu'il y avait derrière les « allures » de l'expérience : «je sais pas du tout ce qui se passe en gym... J'y suis mais je fais illusion, la gym, j'essaie de faire illusion » (Gym, PS2). Les interventions didactiques et les démonstrations corporelles récurrentes semblent ainsi relever d'une tentative d'illusion de la part de P1. Il semble alors que ce soit moins un enjeu didactique qui organise ici son activité didactique qu'une volonté de se sauvegarder en tant qu'enseignant. P1 nous rend alors compte de la division que nous avons soulevée antérieurement: "j'aimerai bien pouvoir leur faire des démonstrations" (Gym, PS1). Il effectue d'ailleurs ces démonstrations. Mais, il nous fait alors l'aveu de sa difficulté à les mener: "en gym, les démonstrations, je suis limité" (Gym, PS1); il s'excuse alors de n'être que de peu d'utilité pour les élèves : "ils sont livrés à eux-mêmes les pauvres en gym » (Gym, PS3). Puis, enfin, lorsqu'on l'interroge sur 
les raisons de son abandon de la gymnastique pour partir faire du handball, il nous répond: "pour m'échapper, je vais pas te le cacher, pour m'échapper " (Gym, PS3). La tentative d'illusion effectuée par P1 n'est réellement que peu tenable et cette difficulté, teintée de culpabilité, le conduit alors à l'abandon.

Nous interprétons alors cette tentative d'illusion et cet abandon de la manière suivante. Nous considérons trois positions face au savoir et à sa transmission qui définissent le sujet didactique. Nous en présentons ici deux d'entre elles et leur articulation. Tout d'abord, le sujet didactique est traversé par une position réelle, c'est-à-dire ce qu'il est réellement en dehors du didactique, mais ce qu'il demeure dans le didactique, par exemple un rugbyman professionnel ou un mauvais danseur. Mais, en classe, le sujet enseignant a aussi une position symbolique à assumer, celle de sujet supposé savoir (Lacan, 1966 ; Chevallard, 1991 ; Terrisse, 1994 ; Buznic-Bourgeacq, 2005). Il est le représentant du savoir dans l'institution classe et les transferts et les demandes des élèves sont dirigés vers cette position symbolique. Ainsi, à défaut d'expérience, la position réelle du sujet, non sachant à l'occasion, va entrer en compromis avec sa position de sujet supposé savoir.

En ce qui concerne P1, la tentative d'illusion constitue alors un processus de sauvegarde de sa position symbolique. Mais, compte tenu de la position réelle de $\mathrm{P} 1$, celle d'un gymnaste inexpérimenté, celui-ci va se retrouver dans un compromis, dans lequel la culpabilité le conduit à l'abandon des démonstrations puis de l'enseignement total. Cet abandon constitue alors l'issue de ce compromis.

\section{3. 2. $\mathrm{P} 4$ : Le refuge des situations : position symbolique et position imaginaire}

23 P4 nous explique les raisons de sa tendance à n'enseigner qu'au travers des aménagements didactiques. Il revient sur son sentiment d'impuissance vis-à-vis de son statut d'enseignant : "je me sentais impuissant... peu efficace... face aux responsabilités de la transmission » (AC). Il nous explique alors pourquoi : «tu vois, je peux pas me réfugier sur un truc que moi je sais» (Danse, PS1). P4 ne peut pas ce réfugier sur son savoir, sur un savoir construit dans son expérience personnelle, pour assumer les responsabilités qui lui incombent par rapport au savoir dans l'institution classe. Il nous explique alors qu'il doit en passer par d'autres moyens : «j'ai du mal à leur dire quelque chose, à leur donner des conseils... donc ça passe par des situations plus adaptées" (AC). On voit donc ici que les aménagements didactiques ne sont pas simplement une manière d'aider les élèves, mais qu'ils constituent plutôt un moyen pour P4 d'assumer le rôle qui lui est délégué par l'institution. D'une même manière, les raisons de son désengagement corporel ne semblent pas être déterminées par des enjeux didactiques. Il nous explique ce qui lui pose problème: "je montrais pas trop, parce que des fois, tu tentes un panier... mais je le sentais pas... si je le rate, je ramasse! Ça le fait pas trop... et bon, en danse, encore pire, rien par rapport à la démonstration » (AC). Si P4 évite de démontrer corporellement, c'est avant tout parce que s'il démontrait, il « ramasserait », c'est-à-dire qu'il subirait une attaque de la part des élèves qui remettrait inévitablement en cause sa position d'enseignant et romprait notamment la relation dissymétrique entre lui et ses élèves par rapport au savoir, puisque l'enseignant est censé être celui qui maitrise par son savoir.

Nous interprétons alors ce refuge derrière les situations et ce désengagement corporel de la manière suivante. Tout d'abord, nous considérons le processus de maintien de la position symbolique comme un organisateur permanent des pratiques. Ainsi, lorsque l'enseignant a une expérience personnelle dans l'activité enseignée, le savoir 
expérientiel lui assure cette position symbolique. En revanche, sans expérience personnelle, il ne peut s'appuyer sur ce savoir expérientiel et il doit trouver d'autres moyens pour assumer sa position. Il nous semble alors que les aménagements didactiques intensifs constituent une voie pour maintenir la position symbolique de l'enseignant dans le didactique à défaut d'expérience. Mais si cette position symbolique peut être relativement maintenue, il y a une troisième position qui définit le sujet didactique plus complexe à assumer sans expérience : la position imaginaire. Lorsque l'enseignant pénètre le milieu de l'élève, c'est sa position imaginaire de maitre qu'il va mettre en jeu, c'est-à-dire de celui qui maîtrise, notamment corporellement, le savoir. Cette position dépasse ainsi la position symbolique dans la mesure où dans le milieu de l'élève, l'enseignant donne à voir une image de lui-même aux élèves. Ainsi, à défaut d'expérience, l'engagement corporel dans le milieu de l'élève peut être relativement faible, car en mettant à l'épreuve une image de maîtrise qu'il ne peut assumer, l'enseignant se risque à une blessure narcissique.

En ce qui concerne $\mathrm{P} 4$, ses enseignements au travers des aménagements didactiques et son désengagement corporel constituent une manière pour lui de conserver sa position symbolique, sans jamais s'engager à mettre à l'épreuve une position imaginaire de maître qu'il ne peut afficher devant les élèves.

Cette analyse interprétative, à l'articulation du clinique et du didactique, nous permet ainsi de porter un autre regard sur le poids de l'expérience personnelle. Car le savoir expérientiel ne s'avance pas seulement dans les pratiques d'enseignement comme la partie épistémique de l'expérience. Il est aussi une construction subjective sur laquelle l'enseignant peut s'appuyer pour assumer sa place dans le didactique. Les aménagements didactiques et les démonstrations corporelles ne sont pas seulement des médias du savoir. Ce sont aussi des moyens pour l'enseignant de se situer dans le didactique. L'expérience personnelle dans l'activité enseignée spécifie alors fortement la place qu'il peut, qu'il désire ou qu'il craint y occuper.

\section{Conclusions : le poids de l'expérience personnelle de l'enseignant}

24 Au travers de nos trois temps d'analyse, nous avons pu dégager progressivement le poids de l'expérience personnelle du professeur d'EPS dans l'activité enseignée sur ses pratiques d'enseignement. En croisant des analyses quantitatives, qualitatives et cliniques, nous avons pu apporter plusieurs éléments de connaissance sur ce poids de l'expérience.

Tout d'abord, nous avons pu identifier des tendances génériques de ce poids. Au terme de ce travail, si l'on s'attache à caractériser les éléments les plus significatifs sans rester dans la catégorisation inhérente à notre cadre d'analyse initial, nous retenons deux éléments. D'une part, le contenu des enseignements avec expérience est beaucoup plus ouvert, dynamique et complexe. Les enseignants ouvrent davantage le contenu en dehors de chaque situation. Ils prennent davantage le temps de formuler ce contenu, de l'extraire de la pratique de l'élève, de le référer à des éléments passés, futurs ou extérieurs à chaque situation. Ce contenu articule davantage d'objets distincts dans des espaces-temps distincts et s'ouvre alors plus sur la complexité de l'APSA. Ils appuient leurs enseignements sur des situations moins didactisées, plus proches des conditions effectives de la pratique globale de l'APSA. Leur contenu est ainsi moins 
bloqué dans les aménagements. D'autre part, les enseignants se situent de manière très différente par rapport au milieu de l'élève. Ils le pénètrent en s'y engageant largement plus avec leur corps. Le corps des enseignants est beaucoup plus source d'émergence du savoir, parfois même uniquement le modèle à suivre. Ils sont ainsi beaucoup plus proches des élèves et l'émergence du contenu s'avère alors beaucoup plus relative à la singularité de chaque situation objective.

Nous avons ensuite pu voir qu'à défaut d'expérience, le contenu des pratiques de deux enseignants singuliers ne s'organisait pas de la même manière. En tentant de construire progressivement le cas de chaque enseignant face au poids de l'expérience, nous avons pu identifier un poids spécifique de l'expérience chez chaque enseignant. Ainsi, sans expérience personnelle, un enseignant va tenter de «faire illusion» en intervenant largement d'un point de vue didactique et en s'engageant corporellement, mais va finalement abandonner son enseignement. Un autre enseignant va se "réfugier» derrière de lourds aménagements didactiques et rester extérieur au milieu de l'élève.

La construction des cas a alors enfin été menée dans une perspective didactique clinique. Nous avons voulu proposer un système d'interprétations prenant en compte les enjeux subjectifs des enseignants pris dans le didactique. En entrant dans le didactique, le sujet enseignant se positionne singulièrement face au savoir et à sa transmission. Nous considérons que les trois positions du sujet didactique que nous avons définies (réelle, symbolique, imaginaire) et leurs articulations sous la forme de compromis constituent une structure organisatrice générique des pratiques d'enseignement. Ainsi, la volonté de maintenir, de sauvegarder, de rechercher ou de fuir ces positions forment un réseau d'enjeux subjectifs qui organisent l'activité didactique du sujet enseignant. Nous avons alors pu voir que l'expérience personnelle, avant de déterminer le contenu des pratiques, pèse sur le sujet enseignant lui-même, sur la place qu'il occupe dans le didactique et sur les processus mis en œuvre pour tenter de l'occuper.

\section{BIBLIOGRAPHIE}

Bachelard, G. (1938). La formation de l'esprit scientifique. Paris : Vrin.

Beillerot, J. (1996). Les savoirs, leurs conceptions, leur nature. In Beillerot, Blanchard-Laville, Mosconi, Pour une clinique du rapport au savoir. Paris : L'Harmattan.

Bourdieu, P. (1980). Le sens pratique. Paris : Les Éditions de Minuit.

Brun, J. (1996). Évolution des rapports entre la psychologie du développement cognitif et la didactique des mathématiques. In J. Brun (Ed.), Didactique des mathématiques. Paris : Delachaux et Niestlé.

Buznic-Bourgeacq, P. (2005). L'expérience de l'enseignant et ses implications didactiques : une étude de cas en EPS. $5^{\text {ème }}$ colloque international Recherche et Formation, Former des enseignants professionnels, savoirs et compétences. IUFM des pays de la Loire. 
Buznic-Bourgeacq, P. (2009). La transmission du savoir expérientiel. Études de cas et analyses comparatives en didactique clinique de l'EPS. Thèse de doctorat, Université de Toulouse.

Buznic-Bourgeacq, P., Terrisse, A., \& Lestel, G. (2008). Expérience personnelle et expérience professionnelle dans l'enseignement de l'EPS : deux études de cas contrastés en didactique clinique. Éducation et didactique (2/3). PUR : Rennes.

Carnus, M.-F., \& Garcia-Debanc, C., Terrisse, A. (2008). Analyse des pratiques des enseignants débutants. Approches didactiques. Grenoble : La pensée sauvage.

Chevallard, Y. (1991). La transposition didactique. Grenoble : La Pensée Sauvage.

Durkheim, E. (1912). Les formes élémentaires de la vie religieuse : le système totémique en Australie. Paris : Alcan.

Juranville, A. (1978). La philosophie comme savoir. In Études philosophiques (avril-juin 1982).

Lalande, A. (1926). Vocabulaire technique et critique de la philosophie. Paris : PUF.

Lacan, J. (1966). Écrits. Paris : Seuil

Merleau-Ponty, M. (1945). Phénoménologie de la perception. Paris : Gallimard.

Piaget, J. (1936). La naissance de l'intelligence chez l'enfant. Neuchâtel : Delâchaux et Niestlé.

Pieron, M. (1998). Compétences de diagnostic et de prescription chez des enseignants de divers degrés d'expertise. In C. Amade-Escot, J.-P. Barrué, J. Bos, P. Dufor, Terrisse (dir.), Recherches en EPS : Bilan et perspectives. Paris, Éditions Revue EPS.

Poussin, G. (2003). La pratique de l'entretien clinique. Paris : Dunod.

Recope, M. (1996). Statut et fonctions su schème de duel dans l'organisation de l'action motrice d'opposition (le cas du Volley-ball). Thèse de doctorat, Université Paris 5.

Schlanger, (1978). Une théorie du savoir. Paris : Vrin.

Sensevy G. (1997). Désir, Institution, Savoirs. In Variations sur une leçon de mathématiques.

L'Harmattan.

Sensevy G., Mercier A., Schubauer-Leoni M.-L. (2000). - Vers un modèle de l'action didactique du professeur à propos de la course à 20. Recherches en didactique des mathématiques, (20/3). Grenoble, La Pensée Sauvage.

Sherrington, C.S. (1933). The Brain and Its Mechanism. Cambridge

Soler, A., \& Durand, M. (1998). La connaissance du contenu chez les enseignants d'EPS. In AmadeEscot, Barrué, Bos, Dufor, Terrisse (dir.), Recherches en EPS : Bilan et perspectives. Paris : Revues EPS.

Terrisse, A. (1994). La question du savoir dans la didactique des APS : essai de formalisation. Note de synthèse pour l'HDR de l'Université Toulouse 3.

Terrisse, A. (2000). Épistémologie de la recherche clinique en sports de combat. In Terrisse (dir.), Recherches en sports de combat et en arts martiaux : état des lieus. Paris : Revues EPS.

Terrisse, A. \& Carnus M.-F. (2009). Didactique de l'éducation physique et sportive. Quels enjeux de savoir? Bruxelles : De Boëck.

Tochon, F.-V. (1996). Grammaire de l'expérience et savoirs-objets : le savoir focal dans la construction de nouveaux modèles de formation. In Barbier (dir .), Savoirs théoriques et savoirs d'action. Paris : PUF.

Varela, F. (1993). L'inscription corporelle de l'esprit. Paris : Le Seuil. 
Vergnaud, G. (1985). Concepts et schèmes dans une théorie opératoire de la représentation. In Psychologie française, 30.

Vergnaud, G. (1987). Les fonctions de l'action et de la symbolisation dans la formation des connaissances chez l'enfant. In J. Piaget, P. Mounoud et J.-P. Bronckart (eds), Encyclopédie de la pléiade (pp. 821-843). Paris : Gallimard.

Vergnaud G. (1989). La formation des concepts scientifiques, relire Vygotsky et débattre avec lui aujourd'hui. In Enfance, (42/1).

Vergnaud G. (1994). L'enfant, la mathématique et la réalité. Berne : Peter Lang

Vergnaud G. (1996). Au fond de l'action, la conceptualisation. In Barbier, Savoirs théoriques et savoirs d'action. Paris : PUF.

Vergnaud G. (2001). Forme opératoire et forme prédicative de la connaissance. In actes du Colloque GDM, Portugais J. (eds), La notion de compétence en enseignement des mathématiques, analyse didactique des effets de son introduction sur les pratiques et sur la formation, Montréal, mai 2001.

Vigarello, G., \& Vives, J. (1996). Technique corporelle et discours technique. In Culture technique, (13).

Wallon, H. (1942). De l'acte à la pensée. Paris : Flammarion.

\section{ANNEXES}

Annexe : tableau III. Résultats quantitatifs

\begin{tabular}{|c|c|c|c|}
\hline & & $\begin{array}{l}\text { Ex } \\
\text { (moyenne) }\end{array}$ & $\begin{array}{l}\text { NEx } \\
\text { (moyenne) }\end{array}$ \\
\hline \multirow{3}{*}{ Dimension conceptuelle } & Diversité invariants & 32,8 & 19,6 \\
\hline & Invariants hiérarchisés & 27,8 & 8,4 \\
\hline & Invariants automatisés & 34 & 6,2 \\
\hline \multirow{2}{*}{ Dimension signifiante } & Diversité signifiants & 104,8 & 66,2 \\
\hline & $\begin{array}{l}\text { Signifiants appartenant à } \\
\text { l'APSA }\end{array}$ & 16,2 & 6,2 \\
\hline \multirow{3}{*}{ Dimension corporelle } & Repères extéroceptifs & 30,4 & 17,6 \\
\hline & Repères proprioceptifs & 41,6 & 19,6 \\
\hline & Démonstrations & 136 & 25,4 \\
\hline \multirow{2}{*}{$\begin{array}{l}\text { Dynamique situationnelle / } \\
\text { représentationnelle }\end{array}$} & $\begin{array}{l}\text { Réinvestissements } \\
\text { d'invariants }\end{array}$ & 32,6 & 21 \\
\hline & Analogies internes & 13,8 & 5,4 \\
\hline
\end{tabular}




\begin{tabular}{|l|l|l|l|}
\hline Part d'initiative & $\begin{array}{l}\text { Tâches sans choix potentiels } \\
\text { (en \%) }\end{array}$ & 57,4 \\
\hline Organisation globale & $\begin{array}{l}\text { Situations globales de } \\
\text { pratique (en \%) }\end{array}$ & 29,2 & 10,8 \\
\hline
\end{tabular}

\section{RÉSUMÉS}

La recherche, dont rend compte cet article, s'inscrit en didactique de l'EPS et interroge le poids de l'expérience personnelle de pratiquant du professeur d'EPS dans une APSA spécifique sur le contenu de l'enseignement effectif de cette dernière (Buznic-Bourgeacq, 2009). Nous présentons alors ici le parcours de cette recherche et les éléments de connaissance qu'elle a pu apporter sur le poids de l'expérience personnelle du professeur d'EPS. L'intention de ce texte est donc double. D'une part, nous identifions les ressorts de l'expérience personnelle dans le didactique à partir d'un cadre théorique original nous permettant d'analyser les pratiques effectives de quatre enseignants débutants dans plusieurs APSA dans lesquelles ils ont des expériences personnelles de pratique très contrastées. D'autre part, nous présentons une démarche de recherche singulière, à l'articulation de la didactique et de la clinique, permettant d'identifier comment l'expérience personnelle pèse sur le sujet enseignant lui-même et sur la place qu'il occupe dans le didactique.

\section{AUTEURS}

\section{PABLO BUZNIC-BOURGEACQ}

DiDiST, CREFI-T (EA 799), Université de Toulouse. Toulouse, France.

\section{ANDRÉ TERRISSE}

DiDiST, CREFI-T (EA 799), Université de Toulouse. Toulouse, France.

\section{ERIC MARGNES}

DiDiST, CREFI-T (EA 799), Université de Toulouse. Toulouse, France. 